\title{
Eficácia de tocilizumabe para pacientes com COVID-19: revisão de literatura
}

\author{
Efficacy of tocilizumab for patients with COVID-19: literature review
}

Eficacia de tocilizumab en pacientes con COVID-19: revisión de la literatura

\section{Alberto Guimarães Medrado Sobrinho ${ }^{1 *}$, Marco Aurélio dos Santos Silva ${ }^{2}$}

Como citar esse artigo. Sobrinho, A.G.B; Silva, M.A.S. Eficácia de tocilizumabe para pacientes com COVID-19: revisão de literatura. Revista Pró-UniverSUS. 2021 Jan./Jun.; 12 (1): $19-26$

\section{Resumo}

Estudos têm revelado que pacientes com COVID-19 tratados com tocilizumabe têm reduzido os níveis de IL-6. Este estudo tem como objetivo realizar uma revisão sobre a eficácia do tocilizumabe no tratamento da COVD-19. Esta revisão contou com artigos publicados nos idiomas português e inglês de janeiro a dezembro de 2020, disponíveis nas bases de dados da Biblioteca Virtual em Saúde (BVS) e PubMed. A busca nas bases de dados resultou em 1191 referências, 502 na Biblioteca Virtual em Saúde (BVS) e 689 na PubMed. Ao serem aplicados os critérios de inclusão e exclusão, 24 estudos preencheram os critérios necessários à realização desta revisão. A única publicação disponível em português foi excluída por se tratar de Diretrizes para o tratamento farmacológico da COVID-19. A maioria dos estudos analisados sugere que o uso de tocilizumabe no tratamento de pacientes em estado grave ou crítico com COVID-19, tem contribuído para melhoria do estado clínico, com redução de citocinas e da resposta inflamatória. Nos estudos em que foram comparados grupos de pacientes com tratamento padrão com grupos de pacientes que receberam tocilizumabe, os resultados também mostram maiores desfechos favoráveis entre os grupos tocilizumabe. Conclui-se que, apesar desses resultados, seriam necessários mais estudos envolvendo um número maior de pacientes para confirmar a eficácia e a segurança do tocilizumabe em pacientes com COVID 19.

Palavras-chave: Coronavirus; Covid-19; Tratamento; Tocilizumabe.

\begin{abstract}
Studies have revealed that patients with COVID-19 treated with tocilizumab have reduced IL-6 levels. This study aims to conduct a review on the effectiveness of tocilizumab in the treatment of VOC-19. This review included articles published in Portuguese and English from January to December 2020, available in the databases of the Virtual Health Library (VHL) and Pubmed. The search in the databases resulted in 1191 references, 502 in the Virtual Health Library (VHL) and 689 in Pubmed. When the inclusion and exclusion criteria were applied, 24 studies met the criteria necessary to carry out this review. The only publication available in Portuguese was excluded because it is Guidelines for the pharmacological treatment of COVID-19. Most of the studies analyzed suggest that the use of tocilizumab in the treatment of patients in severe or critical condition with COVID-19 has contributed to the improvement of clinical status, with reduction of cytokines and inflammatory response. In studies where groups of patients with standard treatment were compared with groups of patients who received tocilizumab, the results also show higher favorable outcomes among the tocilizumab groups. It is concluded that, despite these results, further studies involving a larger number of patients would be necessary to confirm the efficacy and safety of tocilizumab in patients with COVID 19.
\end{abstract}

Keywords: : Coronavirus; Covid-19; Treatment; Tocilizumab.

${ }^{1}$ Acadêmico do Curso de Medicina - Universidade de Vassouras, Vassouras, RJ. Brasil. E-mail: albertogmedrado@gmail.com ORCID: https://orcid.org/0000-0002-6828-0905 ${ }^{2}$ Doutor. Docente do Mestrado Profissional em Ciências Aplicadas em Saúde - Universidade de Vassouras, Vassouras, RJ. Brasil. E-mail: santos-silvabiomec@hotmail.com ORCID: https://orcid.org/0000-0002-2400-6656 


\section{Resumen}

Los estudios han revelado que los pacientes con COVID-19 tratados con tocilizumabe han reducido los niveles de IL-6. El objetivo de este estudio es realizar una revisión de la eficacia de la tocilizumabe en el tratamiento de la COVD-19. Esta revisión contó con artículos publicados en los idiomas portugués e inglés de enero a deciembre de 2020, disponibles en las bases de datos de la Biblioteca Virtual en Salud (BVS) y Pubmed. La búsqueda en las bases de datos resultó en 1191 referencias, 502 en la Biblioteca Virtual en Salud (BVS) y 689 en Pubmed. Al aplicar los criterios de inclusión y exclusión, 24 estudios cumplieron los criterios necesarios para realizar esta revisión. La única publicación disponible en portugués fue excluida por tratarse de Directrices para el tratamiento farmacológico de la COVID-19. La mayoría de los estudios analizados sugieren que el uso de tocilizumabe en el tratamiento de pacientes en estado grave o crítico con COVID-19, ha contribuido a la mejora del estado clínico, con reducción de citoquinas y de la respuesta inflamatoria. En los estudios en los que se compararon grupos de pacientes con tratamiento estándar con grupos de pacientes que recibieron tocilizumabe, los resultados también muestran mayores resultados favorables entre los grupos tocilizumabe. Se concluye que, a pesar de estos resultados, Serían necesarios más estudios con un mayor número de pacientes para confirmar la eficacia y seguridad de tocilizumab en pacientes con COVID 19

Palabras clave: Coronavirus; Covid-19; Tratamiento; Tocilizumab.

\section{Introdução}

A síndrome respiratória aguda grave (SARS$\mathrm{CoV}$ ) e a do Oriente Médio (MERS-CoV) apresentam importante comprometimento do sistema respiratório induzidos por dois coronavirus zoonóticos ${ }^{1}$. Em dezembro de 2019, foi descoberto em Wuhan, China, o novo coronavírus ${ }^{2}$ e a doença associada foi denominada como doença de coronavírus 2019 (COVID-19), declarada como pandemia pela Organização Mundial da Saúde em 11 de março de 2020, devido à crescente morbidade e mortalidade global e que vem provocando desde a sua descoberta ${ }^{3,4}$.

A via de transmissão ocorre principalmente pelo contato de pessoa a pessoa ${ }^{1}$. O diagnóstico da infecção pode ser dividido em clínico, laboratorial e diferencial. A Reação em Cadeia de Polimerase e Transcriptase Reversa (RT- PCR) e métodos sorológicos têm sido os mais utilizados. Em exames radiológicos do tórax, a opacificação em vidro fosco está presente na maioria dos pacientes ${ }^{4}$.

A doença é caracterizada por uma fase inicial de replicação viral que pode ser seguida por uma segunda fase impulsionada pela resposta inflamatória do hospedeiro. Sua apresentação clínica é altamente heterogênea variando de infecção assintomática, sintomas leves ou complicações graves ${ }^{5,6}$.

Nos casos sintomáticos, com infecção leve, o paciente pode apresentar febre, mialgia, dor no corpo, perda do paladar e olfato, não havendo evidência radiológica de pneumonia e a saturação do ar ambiente é > 94\%. Enquanto na infecção moderada há hipóxia com $\mathrm{SpO} 2<94 \%$, há queixa de falta de ar e evidência radiológica de pneumonia moderada ${ }^{7}$.

Os casos graves requerem com frequência a admissão do paciente em unidade de terapia intensiva (UTI), sendo a pneumonia intersticial com insuficiência respiratória a principal causa de necessidade de ventilação mecânica invasiva e morte ${ }^{8,9}$.
A fisiopatologia da infecção grave por SARSCoV-2 tem sido atribuída à síndrome de liberação de citocinas $^{5}$. Pacientes mais críticos podem desenvolver a chamada "tempestade de citocinas", caracterizada pelo aumento da produção de citocinas pró-inflamatórias, resposta inflamatória exacerbada típica da síndrome de liberação de citocinas, podendo produzir danos em longo prazo e fibrose do tecido pulmonar ${ }^{4}, 6$.

Nessa síndrome, as citocinas pró-inflamatórias são liberadas após a ativação da cascata inflamatória, com papel predominante da interleucina-6 (IL-6) ${ }^{5}$. Os níveis plasmáticos elevados de IL-6 parecem estar significativamente associados com a mortalidade entre pacientes de UTI com COVID- $19^{10}$ Amostras de pacientes graves revelaram concentrações plasmáticas aumentadas de IL-6, IL-2, IL-7, IL-10, fator estimulador de colônia de granulócitos (G-CSF), proteína induzível por interferon $\gamma$ (IP10), fator de necrose tumoral $\alpha(2,3$, $4^{11}$, proteína $\mathrm{C}$ reativa (PCR) e ferritina ${ }^{2,3}$.

Esta tempestade de citocinas pode causar uma síndrome do desconforto respiratório agudo (SDRA) com risco de vida em pacientes com pneumonia por SARS CoV-2 ${ }^{12}$. Estudos revelaram que pacientes que sofreram com tempestades de citocinas evoluíram para colapso cardiovascular, disfunção de múltiplos órgãos e morte rápida. Portanto, o bloqueio da produção excessiva de citocinas pode ser de fundamental importância para pacientes com infecção grave ${ }^{2}$.

$\mathrm{Na}$ ausência de um tratamento específico e eficaz para prevenir a progressão dos sintomas respiratórios em pacientes com pneumonia por SARS $\mathrm{CoV}-2$, as abordagens clínicas têm consistido na combinação de medicamentos antivirais e imunomoduladores ${ }^{6,7}$. Os antivirais não têm demonstrado reduções na mortalidade, enquanto os imunomoduladores têm sido uma opção terapêutica possível com base no entendimento fisiopatológico da COVD-199.

Nesse sentido, vêm sendo testados com algum sucesso, diferentes anticorpos monoclonais para 
bloquear o receptor de IL-1 e IL-6 para o tratamento da síndrome de liberação de citocinas ${ }^{7}$. Uma vez que ao neutralizar um fator inflamatório importante, essa molécula pode bloquear a tempestade de citocinas durante o estágio de hiperinflamação sistêmica e reduzir a gravidade da doença ${ }^{13}$.

O Tocilizumabe é um anticorpo monoclonal humanizado recombinante da classe IgG1, que se liga especificamente a receptores solúveis de IL-6 e receptores de IL-6 ligados à membrana, seguido pela inibição da cascata de transdução de sinais próinflamatórios de IL- $6^{5}$. Podendo ser administrado por via intravenosa com base no peso corporal ou como dosagem subcutânea em doses menores ${ }^{4}$. Tocilizumabe é recomendado para tratamento de pacientes com artrite reumatoide, artrite idiopática juvenil sistêmica, artrite de células gigantes e síndrome de liberação de citocinas (SRC), induzida por terapia com células $\mathrm{T}$ de receptor de antígeno quimérico, tem sido considerado uma opção promissora $^{6}{ }^{14}$. Seu uso em pacientes com COVID19 grave tem se mostrado eficaz na melhora da febre, linfopenia, hipoxemia e nos achados tomográficos após o tratamento ${ }^{1}$.

Os resultados dos testes de toxicidade em animais mostraram que o tocilizumabe foi bem tolerado e nenhuma anormalidade significativa foi observada em outros estudos clínico-patológicos ou avaliações histopatológicas ${ }^{1}$. Embora já existam ensaios clínicos buscando uma vacina contra a Covid-19, estamos diante de uma doença altamente contagiosa, com números de casos ainda em ascensão e com milhares de pessoas indo a óbito diariamente, portanto é imperativo que se tenha estratégias de tratamento que possam auxiliar no combate à doença e na recuperação do paciente. Logo, o objetivo do presente estudo foi realizar uma revisão sistemática de literatura sobre a eficácia do tocilizumabe no tratamento de pacientes graves com COVID-19.

\section{Materiais e Métodos}

AFoi realizada uma revisão de artigos publicados de janeiro a dezembro de 2020 nos idiomas português e inglês disponíveis nas bases de dados da Biblioteca Virtual em Saúde (BVS) e PubMed. A pesquisa nas bases de dados foi realizada em 28 de abril de 2021, usando-se a combinação dos descritores: "Coronavírus", "COVID-19" e "tocilizumabe". No filtro das bases de dados foram selecionados: limites humanos, ano 2020, idioma português e inglês, texto completo na BVS e texto completo grátis na PubMed.

Foram incluídos estudos que apresentavam características demográficas como média de idade e gênero dos pacientes; comorbidades associadas, gravidade da doença, terapia com tocilizumabe, evolução clínica, efeitos colaterais e taxas de recuperação e/ou mortalidade. Foram excluídos artigos repetidos, estudos de revisão de literatura, relato de caso, artigos publicados em outros idiomas que não fossem, inglês ou português, fora do período estipulado e que não se enquadrassem ao tema da pesquisa.

\section{Resultados}

A busca nas bases de dados resultou inicialmente em 1191 artigos, sendo 502 na BVS e 689 na PubMed. Seguindo os critérios de seleção e elegibilidade, 24 estudos preencheram os critérios necessários à realização desta revisão. A figura 1 mostra os resultados da busca nas bases de dados.

Quanto ao tipo de estudo dos 24 estudos que integraram esta revisão, destacam-se um estudo

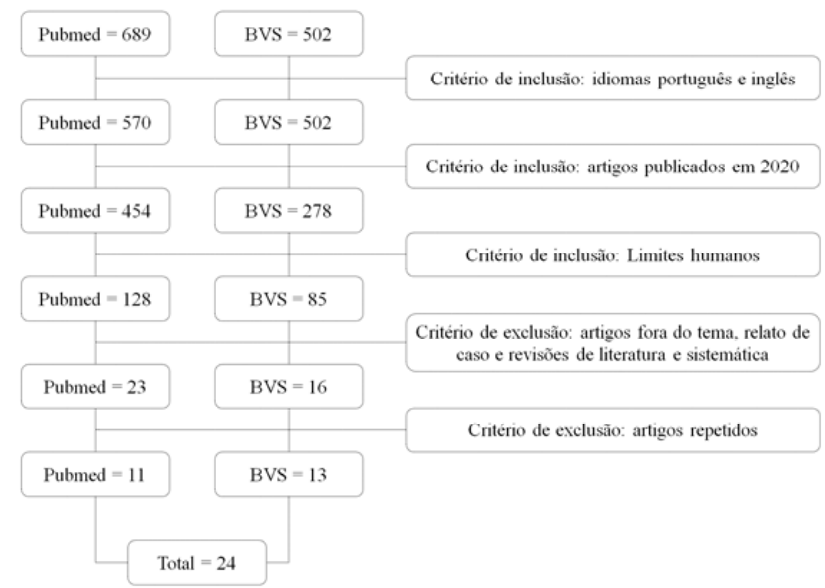

Figura 1. Fluxograma dos resultados da busca bibliográfica nas bases de dados.

randomizado, duplo cego controlado, dois estudos observacional longitudinal, dois estudos retrospectivo de caso controle, dois ensaios clínico prospectivo não controlado, nove estudos retrospectivos, seis estudos de coorte observacional retrospectivo, um estudo coorte e um estudo prospectivo não randomizado.

A população dos 24 estudos envolveu 2152 pacientes com COVID-19, sendo a grande maioria pertencente ao sexo masculino. A média de idade menor foi de 54 anos e a maior de 75,3 anos, destacando que na maioria dos estudos a média de idade dos pacientes se enquadrava entre 61 e 67 anos inclusive.

Todos eram pacientes graves ou críticos com COVD-19, a maioria com uma ou mais comorbidades, onde prevaleciam a hipertensão, diabetes mellitus tipo 2, obesidade, doença pulmonar obstrutiva crônica (DPOC) e doenças cardiovasculares (DCVs).

Em relação ao tratamento, os estudos revelaram uma variariedade de acordo com o tratamento padrão defendido por cada instituição, de forma ampla incluíam 
oxigenoterapia, terapia antiviral com lopinavir/ritonavir ou remdesivir; antibioticoterapia com azitromicina, ceftriaxona, amoxilina; terapia com glicocorticoides, com metilprednisolona e; hidroxicloroquina. O tocilizumabe na maioria dos estudos foi administrado em uma dose IV de $8 \mathrm{mg} / \mathrm{kg}$ usando o peso corporal real com uma dose máxima de $800 \mathrm{mg}$. Ou em duas doses via subcutânea, caso não houvesse resposta clínica favorável.

Quanto aos pacientes tratados com tocilizumabe, os resultados encontrados por cada um dos estudos, efeitos adversos e principais descobertas estão resumidos no Quadro 1.

\section{Discussão}

Em pacientes graves com COVID-19, os níveis de citocinas pró-inflamatórias se encontram muito elevados, concorrendo para piores desfechos clínicos, destacando-se nesse processo hiperinflamatório a IL-6. A hiperinflamação pós-viral com início na segunda semana da doença parece explicar principalmente a gravidade da doença13. Por esta razão, como forma de prevenir ou tratar tempestades de citocinas, têmse recomendado o tratamento com tocilizumabe, um anticorpo monoclonal humanizado contra o receptor de interleucina $\square 6$ (IL $\square$ 6R).

Estudos mostram que após a administração de tocilizumabe, há uma diminuição significativa nos níveis de citocinas, sugerindo uma melhora no estado hiperinflamatório do paciente..$^{9,15}$

Stone e colaboradores ${ }^{18}$ em um ensaio randomizado, duplo cego e controlado por placebo, com 243 pacientes, não forneceram suporte para o conceito de que o bloqueio precoce do receptor de IL-6 é uma estratégia de tratamento eficaz em pacientes hospitalizados com COVID-19 moderado. Foi considerada como hipótese ao estudo que esse bloqueio em pacientes com a doença que ainda não estivessem em ventilação mecânica, interromperia a tempestade de citocinas associado ao COVID-19, evitando casos mais graves. Porém, os resultados desse estudo indicaram que esta intervenção não teve efeito significativo no agravamento da doença.

$\mathrm{Xu}$ e colaboradores ${ }^{1}$ verificaram, em estudo realizado na China, com 21 pacientes, que após a administração de tocilizumabe houve redução na PCR e na suplementação de oxigênio, e que em um espaço de 14 dias após o tratamento a maioria dos pacientes recebeu alta. Malekzadeh e colaboradores ${ }^{17}$ também relatam melhora da necessidade de suporte de oxigênio em pacientes graves nos dias 7 e 14 após administração de tocilizumabe. Outro estudo, também realizado na China, por Luo et $\mathrm{al}^{2}$, com 15 pacientes que receberam tocilizumabe, revelou redução nos níveis de PCR e IL-6. Resultados de estudo realizados na Itália mostraram que em 24 a 72 h após a administração de tocilizumabe em pacientes com insuficiência respiratória rapidamente progressiva, refratária à terapia farmacológica e suporte ventilatório, houve melhora das condições clínicas e respiratórias em $58 \%$ dos pacientes e houve estabilização do quadro clínico em $37 \%{ }^{12}$. Esses resultados sugerem que o tocilizumabe pode reduzir o número de pacientes com pneumonia grave por SARS-CoV-2 em UTI ou a mortalidade entre os pacientes.

Menzella e colaboradores ${ }^{22}$ compararam os valores basais de PCR maior ou igual a $10 \mathrm{mg} / \mathrm{dl}$ e menor que $10 \mathrm{mg} / \mathrm{dl}$ e não observaram qualquer diferença nem para mortalidade nem para necessidade de ventilação mecânica em pacientes tratados com tocilizumabe. Em outro estudo, Mushtaq e colaboradores ${ }^{20}$ relataram um achado importante em relação ao tocilizumabe, que o PCR tem efeito maior do que em comparação com outros marcadores inflamatórios em pacientes que morreram, uma vez que houve redução no PCR e aumento dos marcadores inflamatórios.

Para $\mathrm{Luo}^{2}$ e colaboradores uma única dose de tocilizumabe pode ser eficaz em pacientes graves com IL-6 aproximadamente 10 vezes mais elevada. Nos pacientes em estado moderado com nível elevadíssimo de IL-6, quase 90 vezes o normal, a terapia em duas doses também pode ser benéfica ${ }^{2}$. Outro estudo mostrou que não foi observada incidência de síndrome de liberação de citocinas de grau 4 relacionada a COVID-19 em 1 semana após a administração de uma dose de $162 \mathrm{mg}$ de tocilizumabe por via subcutânea. As tomografias de acompanhamento 7 a 10 dias após o tratamento com tocilizumabe mostraram melhora das manifestações pulmonares em todos os pacientes. ${ }^{4,21}$

Resultados de estudos realizados na Itália mostraram que em 24 a $72 \mathrm{~h}$ após a administração de tocilizumabe em pacientes com insuficiência respiratória rapidamente progressiva, refratária à terapia farmacológica e suporte ventilatório, houve melhora das condições clínicas e respiratórias em 58\% dos pacientes e houve estabilização do quadro clínico em $37 \%{ }^{12}$. Esses resultados sugerem que o tocilizumabe pode reduzir o número de pacientes com pneumonia grave por SARSCoV-2 em UTI ou a mortalidade entre os pacientes. Potere e colaboradores ${ }^{19}$, em uma análise retrospectiva com 20 pacientes, relatam que nenhum dos pacientes tratados com tocilizumabe teve progressão da doença e não apresentaram necessidade de oxigenoterapia ou ventilação invasiva.

Com base nos estudos de caso controle foram obtidos melhores desfechos clínicos entre pacientes que receberam terapia com tocilizumabe do que com o grupo controle, observa-se que essa diferença foi significativa em estudos de Capra $^{8}$ e Klopfenstein ${ }^{13}$.

Em relação às taxas de mortalidade e internação 
Quadro 1. Principais resultados e descobertas dos estudos sobre a administração de tocilizumabe em pacientes graves e críticos com COVID19.

\begin{tabular}{|c|c|c|c|}
\hline AUTOR & $\mathbf{N}$ & OBJETIVO & RESULTADO/DESCOBERTA \\
\hline $\mathrm{Xu}$ et al. ${ }^{1}$ & 21 & $\begin{array}{l}\text { Avaliar a eficácia do tocilizumabe em } \\
\text { pacientes graves com COVID-19 e } \\
\text { buscar uma estratégia terapêutica. }\end{array}$ & $\begin{array}{l}\text { Opacidade da lesão pulmonar foi absorvida em } 90,5 \% \text { dos } \\
\text { pacientes em } 5 \text { dias; } 75 \% \text { dos pacientes reduziram a ingestão } \\
\text { de oxigênio em } 5 \text { dias; nenhum evento adverso foi observado; } \\
100 \% \text { dos pacientes receberam alta em média } 15,1 \text { dias. }\end{array}$ \\
\hline Luo et al. ${ }^{2}$ & 15 & $\begin{array}{l}\text { Apresentar as respostas ao tratamento } \\
\text { de TCZ nos pacientes COVID } \square 19 \mathrm{e} \text {, } \\
\text { em certa medida, fornece orientações } \\
\text { para o uso clínico. }\end{array}$ & $\begin{array}{l}\text { Uma única dose de TCZ parece não melhorar a atividade da do- } \\
\text { ença em pacientes graves, no entanto, doses repetidas (mesmo } \\
\text { repetidas com uma dose menor) podem melhorar a condição de } \\
\text { pacientes criticamente enfermos. }\end{array}$ \\
\hline Kewan et al. ${ }^{3}$ & $\begin{array}{l}51-28 \\
\text { tratados } \\
\text { com TCZ }\end{array}$ & $\begin{array}{l}\text { Relatar nossa experiência com o uso } \\
\text { de tocilizumabe em pacientes hospita- } \\
\text { lizados com COVID - } 19 \text { grave. }\end{array}$ & $\begin{array}{l}\text { O tempo médio para melhora clínica em TCZ vs. sem coortes } \\
\text { de TCZ foi de } 8 \text { dias vs. } 13 \text { dias; Taxas de infecções hospitalares } \\
\text { ocorreram em ambas as coortes ( } 18 \% \text { com TCZ e } 22 \% \text { em ne- } \\
\text { nhuma coorte com TCZ). }\end{array}$ \\
\hline Mastroianni et al. ${ }^{4}$ & 12 & $\begin{array}{l}\text { Realiza um estudo observacional re- } \\
\text { trospectivo de pacientes internados } \\
\text { em hospital com COVID-19 e tra- } \\
\text { tados com tocilizumabe subcutâneo } \\
\text { para investigar a incidência de SRC. }\end{array}$ & $\begin{array}{l}\text { Não foi observada incidência de SRC de grau } 4 \text { em } 1 \text { semana } \\
\text { após a administração de TCZ em todos os } 12 \text { pacientes }(100 \%) \text {; } \\
\text { As tomografias de acompanhamento } 7 \text { a } 10 \text { dias após o tratamen- } \\
\text { to com TCZ mostraram melhora das manifestações pulmonares } \\
\text { em todos os pacientes; Não foram relatados eventos adversos }\end{array}$ \\
\hline Dastan et al. ${ }^{5}$ & 42 & $\begin{array}{l}\text { Avaliar os efeitos do tocilizumab, } \\
\text { como um antagonista da IL- } 6 \text {, em pa- } \\
\text { cientes com infecção grave ou crítica } \\
\text { por SARS-CoV-2. }\end{array}$ & $\begin{array}{l}83,33 \% \text { dos pacientes apresentaram melhora clínica; Nos } 20 \text { ca- } \\
\text { sos graves, apenas } 1 \text { paciente morreu, e entre os } 22 \text { casos críti- } \\
\text { cos, } 6 \text { pacientes }(27 \%) \text { morreram; efeitos adversos neurológicos } \\
\text { foram observados em } 3 \text { pacientes. }\end{array}$ \\
\hline Guaraldi et al.$^{6}$ & $\begin{array}{l}544-179 \\
\text { tratados } \\
\text { com TCZ }\end{array}$ & $\begin{array}{l}\text { Avaliar o papel do tocilizumabe na re- } \\
\text { dução do risco de ventilação mecâni- } \\
\text { ca invasiva e morte em pacientes com } \\
\text { pneumonia COVID-19 grave que re- } \\
\text { ceberam tratamento padrão. }\end{array}$ & $\begin{array}{l}73(20 \%) \text { pacientes no grupo de tratamento padrão morreram, } \\
\text { em comparação com } 13 \text { pacientes tratados com TCZ (seis }[7 \%] \\
\text { tratados por via intravenosa e sete }[8 \%] \text { tratados por via subcu- } \\
\text { tânea). }\end{array}$ \\
\hline Patel et al. ${ }^{7}$ & $\begin{array}{l}20 \text { pa- } \\
\text { cientes }\end{array}$ & $\begin{array}{l}\text { Descrever a eficácia e segurança do } \\
\text { TCZ na pneumonia SARS CoV-2 }\end{array}$ & $\begin{array}{l}\text { O efeito positivo do tratamento na sobrevida com TCZ foi in- } \\
\text { dependente das comorbidades clínicas associadas a resultados } \\
\text { ruins, como idade, diabetes, hipertensão ou doenças cardíacas; } \\
\text { Não houve infecção no grupo TCZ. }\end{array}$ \\
\hline Capra et al.$^{8}$ & 85 & $\begin{array}{l}\text { Avaliar se o tocilizumabe, um anti- } \\
\text { corpo monoclonal anti-receptor solú- } \\
\text { vel da IL-6, reduz a mortalidade dos } \\
\text { pacientes. }\end{array}$ & $\begin{array}{l}92 \% \text { no grupo TCZ se recuperou completamente e teve alta após } \\
\text { uma média de } 12,5 \text { dias (enquanto } 8 \% \text { morreram), enquanto } \\
\text { apenas } 42,1 \% \text { dos pacientes de controle se recuperaram comple- } \\
\text { tamente (enquanto } 57,9 \% \text { morreram); Nenhum efeito colateral } \\
\text { foi relatado. }\end{array}$ \\
\hline Morriison et al. ${ }^{9}$ & 8 & $\begin{array}{l}\text { Determinar as características clíni- } \\
\text { cas associadas à sobrevida hospitalar } \\
\text { de } 28 \text { dias em pacientes com doença } \\
\text { coronavírus } 2019 \text { (COVID-19) que } \\
\text { receberam tocilizumabe. }\end{array}$ & $\begin{array}{l}35,8 \% \text { dos pacientes apresentaram hipertrigliceridemia com } \\
\text { níveis superiores a } 500 \mathrm{mg} / \mathrm{dL} \text {; Infecções secundárias foram } \\
\text { observadas em } 22,2 \% \text { dos pacientes; O recebimento de TCZ } \\
\text { em até } 12 \text { dias após o início dos sintomas pode ser um fator } \\
\text { chave independentemente associado à sobrevida de } 28 \text { dias no } \\
\text { COVID- } 19 \text {. }\end{array}$ \\
\hline Rossotti et al. ${ }^{10}$ & $\begin{array}{l}222 \\
74 \text { trata- } \\
\text { dos com } \\
\text { TCZ }\end{array}$ & $\begin{array}{l}\text { Relatar a segurança e eficácia (em ter- } \\
\text { mos de sobrevida global e alta hospi- } \\
\text { talar) do anti-IL6 tocilizumabe (TCZ) } \\
\text { em indivíduos com COVID-19. }\end{array}$ & $\begin{array}{l}\text { O uso de TCZ foi associado a uma melhor sobrevida global, } \\
\text { mas com uma permanência hospitalar mais longa principalmen- } \\
\text { te devido a eventos adversos bioquímicos, respiratórios e infec- } \\
\text { ciosos; Em indivíduos com doença grave, o TCZ não pareceu } \\
\text { ter nenhum efeito benéfico, embora tenha exercido uma melho- } \\
\text { ra evidente em pacientes críticos. }\end{array}$ \\
\hline Lohse et al. ${ }^{11}$ & 34 & $\begin{array}{l}\text { Identificar fatores prognósticos de } \\
\text { mortalidade em indivíduos tratados } \\
\text { com TCZ para pneumonia grave por } \\
\text { COVID-19 }\end{array}$ & $\begin{array}{l}70 \% \text { dos pacientes com o tratamento com TCZ teve evolução } \\
\text { favorável; Os pacientes que se recuperaram eram ligeiramente } \\
\text { mais jovens - cerca de } 7 \text { anos - do que aqueles nos quais o trata- } \\
\text { mento falhou, embora bons resultados tenham sido obtidos em } \\
\text { vários pacientes com mais de } 85 \text { anos. }\end{array}$ \\
\hline
\end{tabular}


Quadro 1 (cont.). Principais resultados e descobertas dos estudos sobre a administração de tocilizumabe em pacientes graves e críticos com COVID-19.

\begin{tabular}{|c|c|c|c|}
\hline AUTOR & $\mathbf{N}$ & OBJETIVO & RESULTADO/DESCOBERTA \\
\hline Tonati et al. ${ }^{12}$ & 100 & $\begin{array}{l}\text { Descrever a experiência com uma } \\
\text { série de } 100 \text { pacientes consecutivos } \\
\text { com pneumonia COVID-19 grave } \\
\text { tratados com TCZ na Brescia. }\end{array}$ & $\begin{array}{l}\text { Em } 24-72 \mathrm{~h} \text { após a administração de } \mathrm{TCZ}, 58 \% \text { dos pacientes } \\
\text { mostraram uma rápida melhora da condição clínica e respirató- } \\
\text { ria; } 37 \% \text { estabilizaram em comparação com a condição pré- TCZ } \\
\text { em declínio rápido e } 5 \% \text { pioraram (dos quais } 4 \% \text { morreram) }\end{array}$ \\
\hline Klopfenstein et al ${ }^{13}$ & $\begin{array}{l}41-21 \\
\text { tratados } \\
\text { com TCZ }\end{array}$ & $\begin{array}{l}\text { Comparar o resultado, especialmente } \\
\text { admissões em UTI e /ou mortalidade, } \\
\text { entre pacientes com COVID-19 trata- } \\
\text { dos com TCZ e sem TCZ. }\end{array}$ & $\begin{array}{l}\text { Embora os pacientes tratados com TCZ tivessem mais comorbi- } \\
\text { dades e apresentassem formas mais graves, óbito e /ou interna- } \\
\text { ções na UTI foram maiores em pacientes sem TCZ. }\end{array}$ \\
\hline Campochiaro et al.$^{14}$ & $\begin{array}{c}65-32 \\
\text { tratados } \\
\text { com TCZ }\end{array}$ & $\begin{array}{l}\text { Comparar os resultados em } 28 \text { dias de } \\
\text { uma grande coorte de pacientes com } \\
\text { pneumonia COVID-19 grave tratados } \\
\text { com tocilizumabe em adição ao trata- } \\
\text { mento padrão, com aqueles de pacien- } \\
\text { tes concomitantemente hospitalizados } \\
\text { que receberam apenas o tratamento } \\
\text { padrão. }\end{array}$ & $\begin{array}{l}\text { Durante o acompanhamento de } 28 \text { dias, } 69 \% \text { dos pacientes TCZ } \\
\text { experimentaram uma melhora clínica em comparação com } 61 \% \\
\text { dos pacientes de tratamento padrão; A mortalidade foi de } 15 \% \\
\text { no grupo TCZ e } 33 \% \text { no grupo de tratamento padrão; A idade } \\
\text { avançada foi um preditor de morte, enquanto } \mathrm{PaO} 2 \text { : FiO2 basal } \\
\text { mais alta foi um preditor de melhora clínica; A taxa de infecção } \\
\text { e trombose pulmonar foi semelhante entre os dois grupos. }\end{array}$ \\
\hline Antony et al. ${ }^{15}$ & 79 & $\begin{array}{l}\text { Investigar o papel do tocilizumabe } \\
\text { (TCZ) nas tendências de interleucina } \\
\square 6 \text { (IL } \square \text { 6) e VM em pacientes com } \\
\text { SARS } \square \mathrm{CoV} \square 2 \text {. }\end{array}$ & $\begin{array}{l}\text { O uso precoce de TCZ, quando utilizado em pacientes que ne- } \\
\text { cessitam de }>3 \mathrm{~L} \mathrm{O} 2 \text {, reduziu a tempestade de citocinas e a res- } \\
\text { posta inflamatória na maioria dos pacientes. }\end{array}$ \\
\hline Knorr. ${ }^{16}$ & 66 & $\begin{array}{l}\text { Relatar os resultados de pacientes com } \\
\text { COVID } \square 19 \text { grave que receberam to- } \\
\text { cilizumabe em um grande centro mé- } \\
\text { dico urbano nos Estados Unidos. }\end{array}$ & $\begin{array}{l}\text { A melhora clínica foi limitada em pacientes que receberam to- } \\
\text { cilizumabe. Apenas } 15 \% \text { dos pacientes tiveram melhora clínica } \\
\text { em } 7 \text { dias e } 29 \% \text { tiveram melhora clínica em } 14 \text { dias pós-toci- } \\
\text { lizumabe. No geral, } 42 \% \text { dos pacientes que receberam tocilizu- } \\
\text { mabe morreram durante o período de observação. }\end{array}$ \\
\hline Malekzadeh et al. ${ }^{17}$ & 126 & $\begin{array}{l}\text { Avaliar o uso do tocilizumabe subcu- } \\
\text { tâneo em pacientes graves e críticos } \\
\text { com COVID } 19\end{array}$ & $\begin{array}{l}\text { Melhoria do nível de oxigenação em } 7 \text { dias e melhoria dos pa- } \\
\text { râmetros clínicos em } 3 \text { dias após uso da TCZ. Houve redução } \\
\text { do risco de morte, morreram } 6,98 \% \text { dos pacientes graves e } 60 \% \\
\text { dos pacientes críticos. Nenhum evento adverso foi observado. }\end{array}$ \\
\hline Stone et al. ${ }^{18}$ & 243 & $\begin{array}{l}\text { Avaliar a administração da TCZ no } \\
\text { início do curso da doença, para preve- } \\
\text { nir a progressão da COVID } 19\end{array}$ & $\begin{array}{l}\text { A taxa de risco de intubação ou morte no grupo } \mathrm{TCZ} \text { em com- } \\
\text { paração com o grupo placebo foi de } 0,83 \text { e a taxa de risco para } \\
\text { agravamento da doença foi } 1,1 \text {. Os dados mostram que } 18 \% \text { do } \\
\text { grupo TCZ e } 14,9 \% \text { do grupo placebo tiveram pioram da doen- } \\
\text { ça, } 24,6 \% \text { dos pacientes do grupo TCZ e } 21,2 \% \text { do grupo place- } \\
\text { bo ainda estavam necessitando de } \mathrm{O} 2 \text { suplementar. Os pacientes } \\
\text { que receberam TCZ tiveram menos infecções comparados ao } \\
\text { grupo placebo. } 11 \text { pacientes tiveram eventos adversos possivel- } \\
\text { mente relacionados ao TCZ. }\end{array}$ \\
\hline Potere et al. ${ }^{19}$ & 20 & $\begin{array}{l}\text { Avaliar a segurança e eficácia da TCZ } \\
\text { em baixa dose para prevenir pro- } \\
\text { gressão da doença em pacientes com } \\
\text { pneumonia COVID } 19 \text { moderada e } \\
\text { hiperinflamada }\end{array}$ & $\begin{array}{l}\text { Nenhum dos pacientes tratados com TCZ teve progressão da } \\
\text { doença (necessidade de oxigenoterapia ou ventilação mecâni- } \\
\text { ca). Apresentou melhora da oxigenação avaliada pela } \mathrm{P} / \mathrm{F}(11 \% \\
\text { para } 16 \% \text { no primeiro dia) e ( } 23 \% \text { para } 34 \% \text { no terceiro dia). } \\
\text { Sem eventos adversos relevantes. }\end{array}$ \\
\hline Mushtaq et al. ${ }^{20}$ & 40 & $\begin{array}{l}\text { Fornecer evidências através da ex- } \\
\text { periência de um estudo retrospectivo } \\
\text { com uma série de pacientes com CO- } \\
\text { VID } 19 \text { tratados com TCZ }\end{array}$ & $\begin{array}{l}\text { Melhora significativa dos marcadores inflamatórios após a ad- } \\
\text { ministração da TCZ. Dos } 11 \text { pacientes em ventilação mecânica, } \\
7 \text { foram desmamados e extubados, sendo que } 2 \text { falharam. } 77,5 \% \\
\text { (29) dos pacientes apresentaram melhora da necessidade de O2, } \\
\text { enquanto } 79,3 \% \text { (23) desses pacientes apresentou melhora na } \\
\text { radiografia de tórax, sendo que } 28 \text { receberam alta hospitalar } \\
\text { com segurança. } 11 \text { pacientes apresentaram piora, } 9 \text { foram a óbi- } \\
\text { to e } 2 \text { sairam sem orientação médica. }\end{array}$ \\
\hline
\end{tabular}


Quadro 1 (cont.). Principais resultados e descobertas dos estudos sobre a administração de tocilizumabe em pacientes graves e críticos com COVID-19.

\begin{tabular}{|c|c|c|c|}
\hline AUTOR & $\mathbf{N}$ & OBJETIVO & RESULTADO/DESCOBERTA \\
\hline Keske et al. ${ }^{21}$ & 43 & $\begin{array}{l}\text { Apresentar a eficácia e o uso ideal do } \\
\text { tratamento com TCZ com observa- } \\
\text { ções clínicas, laboratoriais e radioló- } \\
\text { gicas }\end{array}$ & $\begin{array}{l}\text { Após a adminitração da TCZ, o tempo médio de internação, } \\
\text { o uso de oxigenoterapia e aduração total de hospitalização foi } \\
\text { menor entre os casos graves do que em casos críticos. Melho- } \\
\text { ra radiológica foi observada em casos graves no sétimo dia de } \\
\text { TCZ. Foram transferidos para a UTI, três casos de } 21 \text { (14\%) dos } \\
\text { pacientes o qual foi administrado TCZ e nenhum foi a óbito. Foi } \\
\text { detectada infecção bacteriana secundária em } 41 \% \text { dos pacientes } \\
\text { internados na UTI. }\end{array}$ \\
\hline Menzella et al. ${ }^{22}$ & 79 & $\begin{array}{l}\text { Analisar a eficácia e segurança do } \\
\text { TCZ em pacientes com COVID } 19 \\
\text { submetidos a ventilação não invasiva }\end{array}$ & $\begin{array}{l}\text { Os níveis de PCR foram significativamente mais baixos } 72 \text { ho- } \\
\text { ras e } 7 \text { dias após o início da terapia com TCZ em comparação ao } \\
\text { grupo de terapia padrão, contudo houve um aumento dos níveis } \\
\text { de IL-6 livre.As probabilidades de óbito ou ser intubado foram } \\
\text { significativamente menores no total de pacientes tratados com } \\
\text { TCZ em aqueles comparados aos não tratados com TCZ. A taxa } \\
\text { de mortalidade geral hospitalar foi de } 38 \% \text {. }\end{array}$ \\
\hline Guillen et al. ${ }^{23}$ & 64 & $\begin{array}{l}\text { Avaliar os efeitos de curto e médio } \\
\text { prazo da terapia precoce com } \mathrm{TCZ}\end{array}$ & $\begin{array}{l}\text { Após a administração daTCZ } 76,6 \% \text { dos pacientes tiveram res- } \\
\text { posta favorável e } 23,4 \% \text { desfavorável ou resultado adverso. } \\
\text { Não foram observadas mortes ou recorrências da doença, } \\
\text { sendo assim a terapia preventiva com TCZ foi segura e asso- } \\
\text { ciada a resultados favoráveis na maioria dos pacientes. }\end{array}$ \\
\hline Tsai et al.,24 & 132 & $\begin{array}{l}\text { Analisar a evolução clínica da mor- } \\
\text { talidade hospitalar em pacientes com } \\
\text { COVID } 19 \text { tratados com TCZ em um } \\
\text { único centro médico }\end{array}$ & $\begin{array}{l}\text { Em relação a mortalidade, houveram } 18 \text { mortes }(27,3 \%) \text { no gru- } \\
\text { po TCZ e } 18 \text { mortes }(27,3 \%) \text { no grupo controle. Foi realizada } \\
\text { uma análise secundária utilizando todo o conjunto de dados, } \\
\text { contendo o escore de propensão como preditor na regressão lo- } \\
\text { gística multivariável, com resultados semelhantes }(\mathrm{p}=0,55)\end{array}$ \\
\hline
\end{tabular}

em UTI, estudos de Guaraldi6, Klopfenstein ${ }^{13}$ e Campochiaro $^{14}$ constataram que foram maiores em pacientes que não receberam tocilizumabe, inclusive entre pacientes com comorbidades clínicas associadas a resultados negativos, como diabetes, hipertensão ou doenças cardíacas.

De acordo com o estudo de Guillén e colaboradores $^{23}$ foi relatado que a falta de resposta do tocilizumabe foi associada a um maior escore Sequentialorgan-failure Assessment (SOFA) e pressão arterial sistólica na admissão e com o índice de comorbidade de Charlson mais alto. Em relação a taxa de mortalidade $(5,6 \%)$, foi demonstrado que os resultados apóiam o início imediato da terapia, sendo seguro a curto e médio prazo e pode estar associado a um desfecho favorável em pacientes hospitalizados $(76,6 \%)$.

Diferentemente desses resultados, um estudo retrospectivo observacional de centro único realizado nos Estados Unidos com 66 pacientes com COVID-19, que estavam em ventilação invasiva ou não invasiva, apenas $15 \%$ tiveram melhora clínica em 7 dias. No geral, $42 \%$ dos pacientes que receberam tocilizumabe morreram dentro de 28 dias. Os autores questionam a eficácia do tocilizumabe no tratamento da COVID$19^{16}$.

Tsai e colaboradores ${ }^{24}$, em um estudo de coorte com 132 pacientes, relatam que não houve diferença na mortalidade entre os pacientes que receberam tocilizumabe e os que receberam apenas suporte.

A ocorrência de efeitos adversos pode ser uma grande preocupação quando se pretende administrar tocilizumabe em pacientes graves, estudos observaram que embora o seu uso esteja associado a uma melhor sobrevida global, há uma permanência hospitalar mais longa principalmente devido a eventos adversos bioquímicos, respiratórios e infecciosos ${ }^{10}$. Observandose ainda a incidência de efeitos neurológicos5. Recomenda-se também o monitoramento contínuo dos níveis de triglicerídeos, uma vez que hipertrigliceridemia foi um evento que ocorreu com frequência entre os pacientes, bem como dos marcadores de pancreatite, transaminases e linfohistiocitose hemofagocítica secundária. ${ }^{9}$

\section{Conclusões}

Níveis elevados de citocinas pró-inflamatórias, principalmente de IL-6, foram apontados como fator mais importante para o agravamento do quadro clínico de pacientes com COVID-19, com comprometimento de diversos órgãos e sistemas, como o pulmão, coração, rins e sistema circulatório, podendo levar a desfechos fatais. 
A maioria dos estudos analisados sugere que o uso tocilizumabe no tratamento de pacientes em estado grave ou crítico com COVID-19 tem contribuído para melhorar o estado clínico, com redução da tempestade de citocinas e da resposta inflamatória.

Nos estudos em que foi comparado grupo de pacientes com tratamento padrão com grupo de pacientes que receberam tocilizumabe, os resultados também mostram maiores desfechos favoráveis entre o grupo tocilizumabe. Porém, apesar desses resultados, seriam necessários mais estudos envolvendo um número maior de pacientes para confirmar a eficácia e a segurança do tocilizumabe em pacientes com COVID 19 envolvendo seus diferentes níveis de gravidade.

\section{Referências}

1. Xu X, Han M, Lia T, Sun W, Wang D, Fu B et al. Effective treatment of severe COVID-19 patients with tocilizumab. Proc Natl Acad Sci U S A. 2020 May 19;117(20):10970-10975

2. Luo P, Liu Y, Qiu L, Liu X, Liu D, Li J et al. Tocilizumab treatment in COVID-19: A single center experience. J Med Virol. 2020;92(7):814-818.

3. Kewan T, Covut F, Al-Jaghbeer MJ, Rose L, Gopalakrishna KV, Akbik B. Tocilizumab for treatment of patients with severe COVID-19: A retrospective cohort study. EClinicalMedicine. 2020 Jun 24;100418.

4. Mastroianni A, Greco S, Apuzzo G, De Santiset S, Oriolo C, Zanolini A, et al. Subcutaneous tocilizumab treatment in patients with severe COVID19-related cytokine release syndrome: An observational cohort study. E Clinical Medicine. 2020 Jul 24;100410.

5. Dastan F, Saffaei A, Haseli S, Marjani M, Moniri A, Abtahian Z, et al. Promising effects of tocilizumab in COVID-19: a non-controlled, prospective clinical trial. Int Immunopharmacol. 2020 Aug 4;06869.

6. Guaraldi G, Meschiari M, Cozzi-Lepri A, Milic J, Tonelli R, Menozzi $\mathrm{M}$, et al. Tocilizumab in patients with severe COVID-19: a retrospective cohort study. Lancet Rheumat. June 24, 2020;8(2):e474-e484.

7. Patel A, Shah K, Dharsandiya M, Patel K, Petel T, Patel M, et al. Safety and efficacy of tocilizumab in the treatment of severe acute respiratory syndrome coronavirus-2 pneumonia: A retrospective cohort study. Indian J Med Microbiol. 2020;38(1):117-123.

8. Capra R, De Rossi N, Mattioli F, Romanelli G, Scarpazza C, Sormani $\mathrm{MP}$, et al Impact of low dose tocilizumab on mortality rate in patients with COVID-19 related pneumonia. Eur J Intern Med. 2020 Jun; 76:31-35.

9. Morrison AR, Johnson JM, Griebe KM, Jones MC, Stine JJ, Hencken $\mathrm{LN}$ et al. Clinical characteristics and predictors of survival in adults with coronavirus disease 2019 receiving tocilizumab. J Autoimmun. $2020 \mathrm{Jul}$ $3 ; 102512$

10. Rossotti R, Travi G, Ughi N, Corradin M, Baiguera C, Fumagalli $R$, et al. Safety and efficacy of anti-il6-receptor tocilizumab use in severe and critical patients affected by coronavirus disease 2019: A comparative analysis. J Infect. 2020; 3(20):30467-9.

11. Lohse A, Klopfenstein T, Balblanc JC, Royer PY, Bossert M, Gendrin $\mathrm{V}$, et al. Predictive factors of mortality in patients treated with tocilizumab for acute respiratory distress syndrome related to coronavirus disease 2019 (COVID-19). Microbes Infect. 2020 Jun 20:S1286-4579(20)30123-4.

12. Toniati P, Piva S, Cattalini M, Garrafa E, Regola F, Castelli F et al. Tocilizumab for the treatment of severe COVID-19 pneumonia with hyperinflammatory syndrome and acute respiratory failure: A single center study of 100 patients in Brescia, Italy. Autoimmun Rev. Jul 2020; 19(7):102568

13. Klopfenstein T, Zayet S, Lohse A, Balblanc JC, Badie J, Royer PY, et al . Tocilizumab therapy reduced intensive care unit admissions and/or mortality in COVID-19 patients. Med Mal Infect. 2020 Aug; 50(5):397-
400.

14. Campochiaro C, Della-Torre E, Cavalli G, De Luca G, Ripa M, Boffini N, et al;. Efficacy and safety of tocilizumab in severe COVID-19 patients: a single-centre retrospective cohort study. Eur J Intern Med. 2020 Jun; 76:43-49.

15. Antony SJ, Davis MA, Davis MG, Almaghlouth NK, Guevara R, Omar F, et al. Early use of tocilizumab in the prevention of adult respiratory failure in SARS-CoV-2 infections and the utilization of interleukin-6 levels in the management. J Med Virol. $2020 \mathrm{Jul}$; 9:10.

16. Knorr JP, Colomy V, Mauriello CM, Ha S. Tocilizumab in patients with severe COVID-19: A single-center observational analysis. J Med Virol. 2020 Jun; 17:10

17. Malekzadeh R, Abedini A, Mohsenpour B, Sharifipour E, Ghasemian $\mathrm{R}$, Javad-Mousavi SA, et al. Subcutaneous tocilizumab in adults with severe and critical COVID-19: A prospective open-label uncontrolled multicenter trial. Int Immunopharmacol. 2020 Oct 13; 107102.

18. Stone JH, Frigault MJ, Serling-Boyd NJ, Fernandes AD, Harvey L, Foulkes AS, et al. Efficacy of tocilizumab in patients hospitalized with Covid-19. N Engl J Med 2020 Dec 10; 383:2333-2344

19. Potere N, Nisio MD, Rizzo G, Vella ML, Polilli E, Agostinone A, et al. Low-dose subcutaneous tocilizumab to prevent disease progression in patients with moderate COVID-19 pneumonia and hyperinflammation. Int J of Infectious Diseases 1002020 Jul 23; 421-424.

20. Mushtaq MZ, Mahmood SBZ, Jamil B, Aziz A, Ali AS, Outcome of COVID-19 patients with use of Tocilizumab: A single Center experience.Int Immunopharmacol. 2020 Aug 28; 106926

21. Keske S, Tekin S, Sait B, Irkoren P, Kapmaz M, Çimen C, et al. Appropriate use of tocilizumab in COVID 19 infection. Int J of Infectious Diseases 99. 2020 Jul 21; 338-343.

22. Menzella F, Fontana M, Salvarani C, Massari M, Ruggiero P, Scelfo C, et al. Efficacy of tocilizumab in patients with COVID-19 ARDS undergoing noninvasive ventilation. Critical Care, 2020; 24(1):1-9.

23. Guillén L, Padilla S, Fernández M, Agulló V, Garcia JÁ, Telenti G, et al. Preemptive interleukin-6 blockade in patients with COVID-19. Scientific Reports. 2020; 10(1):1-9

24. Tsai A, Diawara O, Nahass RG, Brunetti L. Impact of tocilizumab administration on mortality in severe COVID-19. Scientific Reports 2020; 10(1): $1-7$ 